\title{
ON THE COMPUTATION OF THE CAUCHY INDEX*
}

\author{
BY BRIAN D. O. ANDERSON (University of Newcastle, Australia)
}

\begin{abstract}
The Cauchy index of a real rational function can be computed by evaluating the signature of a certain Hankel matrix. Alternative procedures for its computation are presented here, one of which offers greater computational simplicity.

1. Introduction. Let $p(x)$ and $q(x)$ be relatively prime polynomials with real coefficients. The Cauchy index over $(-\infty, \infty)$ of $p(x) / q(x)$, written $I_{-\infty}^{+\infty}(p(x) / q(x)$, is defined as the number of jumps from $-\infty$ to $+\infty$ less the number of jumps from $+\infty$ to $-\infty$ of the fraction $p(x) / q(x)$ when $x$ varies from $-\infty$ to $+\infty[1]$.

Of course, it is possible to evaluate $I_{-\infty}^{+\infty}(p(x) / q(x))$ by determining the real zeros of $q(x)$ and the associated residues of $p(x) / q(x)$ at these real zeros. But the efficiency of such a procedure is clearly tied to the case with which real roots of a polynomial can be found. An alternative and more appealing procedure for the evaluation of $I_{-\infty}^{+\infty}(p(x) / q(x))$ is discussed in [1]; it is based on computing the signature of a Hankel matrix constructed from the Markov parameters associated with $p(x) / q(x)$. (Precise definitions are given later.)
\end{abstract}

Our aim here is to give two alternative characterizations of the Cauchy index; one is especially attractive from the computational point of view as it avoids construction of the Markov parameters or indeed any rational functions of the coefficients of $p(x)$ and $q(x)$; it does, however require computation of integral, as opposed to rational, functions of the coefficients. The second characterization leads to an infinity of matrices whose signature defines the Cauchy index of $p(x) / q(x)$. Amongst this family is the matrix of integral functions of the coefficients.

As a preliminary simplification, we demand that the degree of $p(x)$ be less than that of $q(x)$. If this is initially not the case, we can of course write

$$
p(x) / q(x)=r(x)+p^{\prime}(x) / q(x),
$$

where $p^{\prime}(x)$ and $r(x)$ are polynomials, with the degree of $p^{\prime}(x)$ less than that of $q(x)$. Then

$$
I_{-\infty}^{+\infty}(p(x) / q(x))=I_{-\infty}^{+\infty}\left(p^{\prime}(x) / q(x)\right) .
$$

2. The Hankel matrix of Markov parameters. The Markov parameters $s_{i}, i=0$, $1,2, \cdots$, associated with $p(x) / q(x)$ are defined as follows, where we now assume that the degree of $p(x)$ is less than that of $q(x)$ :

$$
p(x) / q(x)=s_{0} / x+s_{1} / x^{2}+s_{2} / x^{3}+\cdots .
$$

* Received April 8, 1970; revised version received May 21, 1971. Work supported by Australian Research Grants Committee, Australian-American Educational Foundation in Australia and USAF Contract F44620-68-C-0023 of the Air Force Office of Scientific Research. Part of this work was performed while the author was a Visiting Professor in the Information and Control Sciences Center, Institute of Technology, Southern Methodist University, Dallas, Texas 75222, U.S.A. Sincere thanks are extended to Professor Andrew P. Sage for his hospitality at this time. 
Note that the region of convergence of the right-hand side of (2.1) is irrelevant in defining the $s_{i}$. The associated infinite Hankel matrix is

$$
H=\left[\begin{array}{cccccc}
s_{0} & s_{1} & s_{2} & \cdot & \cdot & \cdot \\
s_{1} & s_{2} & \cdot & \cdot & \cdot & \cdot \\
s_{2} & \cdot & \cdot & \cdot & \cdot & \cdot \\
\cdot & \cdot & \cdot & \cdot & \cdot & \cdot \\
\cdot & \cdot & \cdot & \cdot & \cdot & \cdot
\end{array}\right] .
$$

Let $H_{m m}$ denote the matrix consisting of the first $m$ rows and columns of $H$.

In [1], the following is established.

THEOREM 1. Suppose $q(x)$ has degree $n$. Then for all $m \geq n$, rank $H_{m m}=\operatorname{rank} H_{n n}$ and signature $H_{m m}=$ signature $H_{n n}=I_{-\infty}^{+\infty}(p(x) / q(x))$.

Thus to evaluate the Cauchy index, it is necessary to determine the first $2 n-1$ Markov parameters associated with $p(x) / q(x)$, then to form $H_{n n}$ consisting of the first $n$ rows and columns of $H$ in (2.2), and finally to determine the signature of $H_{n n}$. Of course, the signature of $H_{n n}$ can be found by examining the signs of the leading minors [1].

3. Matrix of integral functions of polynomial coefficients. Let the polynomials $p(\cdot)$ and $q(\cdot)$ be defined by

$$
\begin{aligned}
& p(x)=b_{0} x^{m}+b_{1} x^{m-1}+\cdots+b_{m}, \\
& q(x)=a_{0} x^{n}+a_{1} x^{n-1}+\cdots+a_{n},
\end{aligned}
$$

with $n>m$. From these polynomials $p(\cdot)$ and $q(\cdot)$, we define coefficients $c_{i}$ by the Bezoutian form

$$
\frac{p(y) q(x)-q(y) p(x)}{x-y}=\sum_{i, j=1}^{n} c_{i j} x^{i-1} y^{i-1} .
$$

Clearly, $c_{i i}=c_{i i}$. Below we shall prove the following result.

TheORem 2. Let $C=\left(c_{i j}\right)$, where the entries $c_{i j}$ are formed as indicated above. Then signature $C$ is the Cauchy index over $(-\infty, \infty)$ of $p(x) / q(x)$.

First, let us note a simple expression for the coefficients $c_{i j}$ in terms of the coefficients of the polynomials $p(\cdot)$ and $q(\cdot)$. From (3.2), it follows by multiplying on the left and right by $x-y$ and equating coefficients of like powers that

$$
a_{n-i} b_{m-i}-a_{n-j} b_{m-i}=c_{i j+1}-c_{i+1 ;} .
$$

From this identity we obtain

$$
c_{i j}=\sum_{k \geq 0}\left(a_{n-i-k} b_{m-j+1+k}-a_{n-j+1+k} b_{m-i-k}\right)
$$

where we take $a_{l}=0$ if $l<0$ or $l>n$ and $b_{l}=0$ if $l<0$ or $l>m$.

Proof of Theorem 2. Recalling that, formally,

$$
p(x)=q(x)\left[\frac{s_{0}}{x}+\frac{s_{1}}{x^{2}}+\cdots\right]
$$


we have

$$
\begin{aligned}
\sum c_{i j} x^{i-1} y^{i-1} & =\frac{p(y) q(x)-q(y) p(x)}{x-y} \\
& =\frac{q(x) q(y)}{x y} \frac{1}{(1 / y)-(1 / x)}\left[s_{0}\left(\frac{1}{y}-\frac{1}{x}\right)+s_{1}\left(\frac{1}{y^{2}}-\frac{1}{x^{2}}\right)+\cdots\right] \\
& =\frac{q(x) q(y)}{x y}\left[s_{0}+s_{1}\left(\frac{1}{y}+\frac{1}{x}\right)+s_{2}\left(\frac{1}{y^{2}}+\frac{1}{y x}+\frac{1}{x^{2}}\right)+\cdots\right] \\
& =q(x)\left[\frac{1}{x} \frac{1}{x^{2}} \frac{1}{x^{3}} \cdots\right] H\left[\frac{1}{y} \frac{1}{y^{2}} \frac{1}{y^{3}} \cdots\right]^{\prime} q(y),
\end{aligned}
$$

where the superscript prime denotes matrix transposition.

Now write $q(x) / x^{i}$ as $q_{i}(x)+r_{i}(x)$ where $q_{i}(x)$ is a polynomial in $x$, and find $r_{i}(x)$ polynomial in $1 / x$ with no constant terms. Thus, for example, $q_{1}(x)=a_{0} x^{n-1}+\cdots+a_{n-1}$ and $r_{1}(x)=a_{n} / x$. Then (3.4) yields

$\sum c_{i j} x^{i-1} y^{j-1}=\left[q_{1}(x)+r_{1}(x) q_{2}(x)+r_{2}(x) \cdots\right] H\left[q_{1}(y)+r_{1}(y) q_{2}(y)+r_{2}(y) \cdots\right]^{\prime}$.

Since the left side has no terms in $1 / x$ or $1 / y$ it follows that

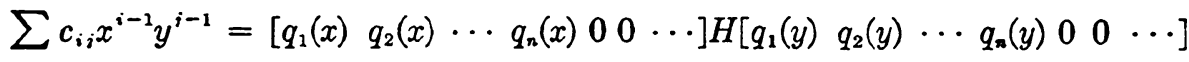

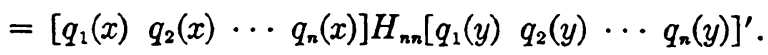

It is readily verified that

$$
\left[q_{1}(x) q_{2}(x) \cdots q_{n}(x)\right]=\left[\begin{array}{llll}
1 & x & \cdots & x^{n-1}
\end{array}\right]\left[\begin{array}{ccccc}
a_{n-1} & a_{n-2} & \cdots & a_{1} & a_{0} \\
a_{n-2} & a_{n-3} & \cdots & a_{0} & 0 \\
\cdot & \cdot & & \cdot & \cdot \\
\cdot & \cdot & & \cdot & \cdot \\
a_{1} & a_{0} & \cdots & 0 & 0 \\
a_{0} & 0 & \cdots & 0 & 0
\end{array}\right],
$$

and so with

$$
A=\left[\begin{array}{ccccc}
a_{n-1} & a_{n-2} & \cdots & a_{1} & a_{0} \\
a_{n-2} & a_{n-3} & \cdots & a_{0} & 0 \\
\cdot & \cdot & & \cdot & \cdot \\
\cdot & \cdot & & \cdot & \cdot \\
a_{1} & a_{0} & \cdots & 0 & 0 \\
a_{0} & 0 & \cdots & 0 & 0
\end{array}\right]
$$

Eqs. (3.5), (3.6) and (3.7) imply $C=A^{\prime} H_{n n} A$. The rank and signature of $C$ are the same as the rank and signature of $H_{n n}$ in view of the obvious nonsingularity of $A$. Application of Theorem 1 then yields the desired result.

4. A class of matrices yielding the Cauchy index. We shall make use of the language of linear system theory [2]. As earlier, we assume $p$ and $q$ relatively prime, $p$ of degree $m$ and $q$ of degree $n>m$. Let $F$ be an $n \times n$ matrix and let $g$ and $h$ be $n$-vectors with the 
entries of $F, g$ and $h$ all real. Then the triple $\{F, g, h\}$ is a minimal realization of $p(x) / q(x)$ if

$$
p(x) / q(x)=h^{\prime}(x I-F)^{-1} g .
$$

Minimal realizations always exist-in fact there is an infinity of minimal realizations associated with the rational function $p(x) / q(x)$.

Our main result is as follows.

Theorem 3. Let $F, g, h$ be a minimal realization of $p(x) / q(x)$ in the above sense. Then there exists a unique symmetric matrix $P$ satisfying

$$
P F=F^{\prime} P, \quad P g=h,
$$

and signature $P$ is $I_{-\infty}^{+\infty}(p(x) / q(x))$. Further, $P$ is computable by simple algebraic operations which exclude polynomial factoring or its equivalent.

Proof. (i) Computation. From (4.2) we have

$$
P F g=F^{\prime} P g=F^{\prime} h, \quad P F^{2} g=F^{\prime 2} P g=\left(F^{\prime}\right)^{2} h,
$$

and in fact $P\left[g F g \cdots F^{m-1} g\right]=\left[h F^{\prime} h \cdots\left(F^{\prime}\right)^{n-1} h\right]$ or

$$
P V=W
$$

with obvious definitions of $V$ and $W$. The matrix $V$ is invertible because $\{F, g, h\}$ is minimal and thus completely controllable [2]. Therefore, $P=W V^{-1}$.

We must also verify that (4.3) implies (4.2). The second equation of (4.2) follows by equating the first column on each side of (4.3). To check the first equation of (4.2), we proceed as follows. By the Cayley-Hamilton theorem (4.3) implies $P e^{F t} g=e^{F{ }^{\prime}} h$. Differentiate to obtain $P F e^{F t} g=F^{\prime} e^{F^{\prime} t} h=F^{\prime} P e^{F t} g$. So $\left(P F-F^{\prime} P\right) e^{F t} g=0$, and an application of complete controllability [2] yields that $P F=F^{\prime} P$.

(ii) Symmetry. Observe that $V^{\prime} P V=V^{\prime} W^{\prime}$ is symmetric, because the $i-j$ term of $V^{\prime} W$ is $g^{\prime}\left(F^{i-1}\right)^{\prime}\left(F^{\prime}\right)^{i-1} h=h^{\prime} F^{i+i-2} g$.

(iii) Uniqueness. Eqs. (4.2) and (4.3) are equivalent and (4.3) has a unique solution.

(iv) Signature property. Since $V$ is nonsingular, signature $P=$ signature $V^{\prime} P V^{\prime}=$ signature $V^{\prime} W$ by (4.3). The $i-j$ term of $V^{\prime} W$ is $h^{\prime} F^{i+j-2} g$ which can be verified, using (3.1), to be $s_{i+i-2}$. Hence $V^{\prime} W$ is precisely $H_{n n}$, the first $n$ rows and columns of the infinite Hankel matrix defined by the Markov parameters of $p(x) / q(x)$. Theorem 1 yields the desired result.

We shall show now that one particular minimal realization of $p(x) / q(x)$ yields a matrix $P$ which is, to within a positive constant multiplier, precisely the matrix $C$ of Sec. 2 .

It may be verified by direct calculation, and it is shown in [2], that with $p(x)$ and $q(x)$ defined as in (3.1), a minimal realization of $p(x) / q(x)$ is provided by

$$
F=\left[\begin{array}{ccccc}
0 & 1 & 0 & \cdots & 0 \\
0 & 0 & 1 & \cdots & 0 \\
\vdots & & & \ddots & \\
-\frac{a_{n}}{a_{0}} & -\frac{a_{n-1}}{a_{0}} & & \cdots & -\frac{a_{1}}{a_{0}}
\end{array}\right] g=\left[\begin{array}{c}
0 \\
0 \\
\vdots \\
1
\end{array}\right] h=\left[\begin{array}{c}
\frac{b_{m}}{a_{0}} \\
\frac{b_{m-1}}{a_{0}} \\
\vdots
\end{array}\right]
$$


Theorem 4. Let $p(x)$ and $q(x)$ be defined as in (3.1), and let a minimal realization of $p(x) / q(x)$ be as given in (4.4). Then the solution $P$ of $E q s$. (4.2) is $P=a_{0}^{-2} C$, where $C=\left(c_{i j}\right)$ with $c_{i j}$ defined by (3.3).

To prove the theorem, we make use of the following lemma, the proof of which is immediate, by direct verification.

Lemma. Let $F, g$ and $h$ be as in (4.4), and let $\pi(x)=\left[1 x x^{2} \cdots x^{n-1}\right]^{\prime}$. Then $F \pi(x)=$ $x \pi(x) \bmod q(x)$ and $h^{\prime} \pi(x)=p(x) / a_{0}$.

Proof of Theorem 4. From (3.2) and the definitions of $C$ and $\pi(x)$ we have

$$
\frac{p(y) q(x)-p(x) q(y)}{x-y}=\sum_{i, j=1}^{n} c_{i} x^{i-1} y^{i-1}=\pi^{\prime}(x) C \pi(y)
$$

and so

$$
p(y) q(x)-p(x) q(y)=x \pi^{\prime}(x) C \pi(y)-\pi^{\prime}(x) C \pi(y) y .
$$

Now reduce both sides modulo $q(x)$ and then modulo $q(y)$. The left side becomes zero, while the right side may be evaluated using the lemma to yield $\pi^{\prime}(x)\left[F^{\prime} C-C F\right] \pi(y)=0$. Since this holds for all $x$ and $y$,

$$
C F=F^{\prime} C
$$

Text, observe that in Eq. (4.5) the coefficient of $y^{n}$ on the left-hand side is $-a_{0} p(x)=$ $-a_{0}^{2} h^{\prime} \pi(x)$ by the lemma. The coefficient on the right side is evidently $-\pi^{\prime}(x) C g$. Consequently $\pi^{\prime}(x)\left[C g-a_{0}^{2} h\right]=0$, whence

$$
C g=a_{0}^{2} h
$$

Now the uniqueness of $P$ and comparison of (4.2), (4.6) and (4.7) yield the result $C=a_{0}^{2} P$.

5. Concluding remarks. Various special choices of $p(x)$ and $q(x)$ lead to the Cauchy index of $p(x) / q(x)$ being the number of real roots of a polynomial, or the number of right half plane zeros of a polynomial. In the latter case, the matrix $C$ of Sec. 3 becomes identical with the Hermite matrix [3], [4] whose relation to the matrix $H_{n n}$ of Sec. 2 has been explored in [5]. A theorem similar to Theorem 4 has been derived in [6], where special choices of $p(\cdot)$ and $q(\cdot)$ enable counting of the zeros of a polynomial inside the unit circle. We note too the use of Bezoutian form of (3.2) in [7] to compute the degree of the highest common factor of $p(x)$ and $q(x)$.

The Cauchy index can also be computed from the signs of the Hurwitz determinants. Their relation to the signs of a sequence of nested determinants of the Hankel matrix appears in [8] and [9], and to the signs of a sequence of nested determinants of the Bezoutian matrix $C$ in [7].

\section{REFERENCES}

[1] F. R. Gantmacher, The theory of matrices, Chelsea, New York, 1959

[2] L. A. Zadeh and C. A. Desoer, Linear system theory, McGraw-Hill, New York, 1963

[3] C. Hermite, Sur le nombre des racines d'une equation algébrique comprise entre des limites données, J. Reine Angew. Math. 52, 39-51, (1854) and Oeuvres 1, pp. 397-414

[4] P. C. Parks, A new proof of the Routh-Hurwitz stability criterion using the second method of Lyapunov, Proc. Cambridge Philos. Soc. 58, part 4, pp. 694-702 (1962)

[5] B. D. O. Anderson, Application of the second method of Lyapunov to the proof of the Markov stability criterion, Internat. J. Control. 5, 473-482 (1967) 
[6] R. E. Kalman, On the Hermite-Fujiwara theorem in stability theory, Quart. Appl. Math. 23, 279-282 (1965)

[7] A. S. Householder, Bezoutiants, elimination and localization, SIAM Rev. 12, 73-78 (1970)

[8] W. Jarominek, Investigating linear systems of automatic control by means of determinant stability margin indices, Proc. First IF AC Congress 1, 76-84, Butterworths, London, 1961

[9] W. Jarominek, The equivalence of the Routh-Hurwitz and the Markov stability criteria, Soviet Physics Dokl. 5, 78-82 (1960) 